\title{
Design of Coupled Resonators Bandpass Filter with Defected Ground Structure
}

\author{
Gi-Rae Kim, Member, KIMICS
}

\begin{abstract}
In this paper a four-pole elliptic function bandpass filter is designed with two ground slots. A research of microstrip bandpass filters (BPF) using defected ground structures (DGS) is presented. DGS technique allows designs of tight couplings without the necessity of using very narrow coupling gaps. The simulator Sonnet is used to design the resonator and to calculate the coupling coefficient of the basic coupling structure. Compared to similar microstrip filters without defected ground structure, the simulated performances of these novel structures indicate some technological advantages.
\end{abstract}

Index Terms - compact coupled resonators, bandpass filer (BPF), defected ground structure (DGS).

\section{INTRODUCTION}

IN wireless communication systems, small size and high performance filters are needed to reduce the cost and improve the system performance. They can be designed in many different ways. However, further miniaturization becomes more difficult for this filter. Planar filters provide good miniaturization ability [1-4]. Therefore, there has been much research conducted on planar filters and their components.

Since microstrip resonators are the basic components of a planar filter design, it is necessary to select proper resonator types used in a filter design. A conventional haft-wavelength open-line microstrip resonator is too large to be used in the modern communication system such as $900 \mathrm{MHz}, 1800 \mathrm{MHz}$ for personal communication systems (PCS). The hairpin filters [1, 5-7] were folded from the open line wavelength microstrip resonator to become U-shaped resonators and make progress in circuit size reduction from the parallel-coupled line structure. Ground slots have many applications in microwave techniques. Slot antennas and slot coupled antennas [8] have been continuously developed and are widely used in communications. The slot coupling is a convenient way to couple microstrip lines in multilayer circuits [9].

In this paper are presented investigations on the effects of ground slots on the couplings between hairpin resonators. Ground slots in the ground plane can enhance the electric coupling, or the electric part of a mixed

Manuscript received March 10, 2011; revised March 26, 2011; accepted April 9, 2011.

Gi-Rae Kim is with the Department of Electronics Engineering, Silla University, Busan, 617-736, Korea, (Email: grkim@silla.ac.kr) coupling between two adjacent resonators. The results of these investigations were used in the design of some fourpole cross coupled planar microwave bandpass Filters with a pair of attenuation poles at imposed finite frequencies. The Filters were designed with two ground slots.

\section{FILTER CHARACTERISTICS}

The filter having only one pair of transmission zeros (or attenuation poles) at finite frequencies gives much improved skirt selectivity, making it a viable intermediate between the Chebyshev and elliptic-function filters, yet with little practical difficulty of physical realization. The transfer function of this type of filter is

$$
\begin{gathered}
\left|S_{21}(\Omega)\right|^{2}=\frac{1}{1+\varepsilon^{2} F_{n}^{2}(\Omega)} \\
\varepsilon_{r}=\frac{1}{\sqrt{10^{-\frac{L_{R}}{10}}-1}} \\
F_{n}(\Omega)=\cosh \left\{(n-2) \cosh ^{-1}(\Omega)+\cosh ^{-1}\left(\frac{\Omega_{\Omega} \Omega-1}{\Omega_{a}-\Omega}\right)+\cosh ^{-1}\left(\frac{\Omega_{a} \Omega+1}{\Omega_{a}+\Omega}\right)\right\}
\end{gathered}
$$

where $\Omega$ is the frequency variable that is normalized to the passband cut-off frequency of the lowpass prototype filter, $\varepsilon_{\mathrm{r}}$ is a ripple constant related to a given return loss $\mathrm{L}_{\mathrm{R}}=20 \log |\mathrm{S} 11|$ in $\mathrm{dB}$, and $\mathrm{n}$ is the degree of the filter. It is obvious that $\Omega= \pm \Omega_{\mathrm{a}}\left(\Omega_{\mathrm{a}}>1\right)$ are the frequency locations of a pair of attenuation poles. Note that if $\Omega_{\mathrm{a}} \rightarrow \infty$ the filtering function $F_{n}(\Omega)$ degenerates to the familiar Chebyshev function. The transmission frequency response of the bandpass filter may be determined using frequency mapping, i.e.,

$$
\Omega=\frac{1}{F B W} \cdot\left(\frac{\omega}{\omega_{0}}-\frac{\omega_{0}}{\omega}\right)
$$

in which $\omega$ is the frequency variable of bandpass filter, $\omega_{0}$ is the mid-band frequency and FBW is the fractional bandwidth. The locations of two finite frequency attenuation poles of the bandpass filter are given by 


$$
\begin{gathered}
\omega_{a 1}=\omega_{0} \frac{-\Omega_{a} F B W+\sqrt{\left(\Omega_{a} F B W\right)^{2}+4}}{2} \\
\omega_{a 2}=\omega_{0} \frac{\Omega_{a} F B W+\sqrt{\left(\Omega_{a} F B W\right)^{2}+4}}{2}
\end{gathered}
$$

\section{COUPLING CONFIGURATIONS}

The microstrip circuit was designed on a Teflon dielectric substrate, with a thickness of $0.7874 \mathrm{~mm}$, a dielectric constant $\varepsilon_{\mathrm{r}}$ of 2.17. In order to develop applications for the $1.8 \mathrm{GHz}$ frequency band, the sizes of microstrip hairpin resonators were confirmed. The hairpin resonators size is: $\mathrm{W}=5.5 \mathrm{~mm}, \mathrm{~L}=23.7 \mathrm{~mm}, \mathrm{~S}=18 \mathrm{~mm}$. The ground slots are all rectangular, with different lengths $1_{\text {slot }}$ and widths $\mathrm{w}_{\text {slot. }}$ The geometries of the main coupling configurations are shown in Figs. 1.

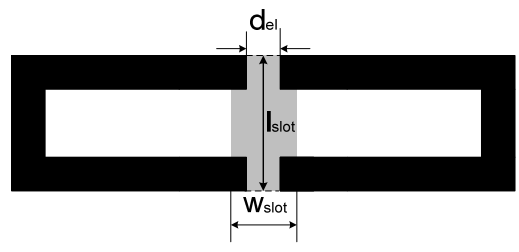

Fig. 1(a). Electric coupling configuration.

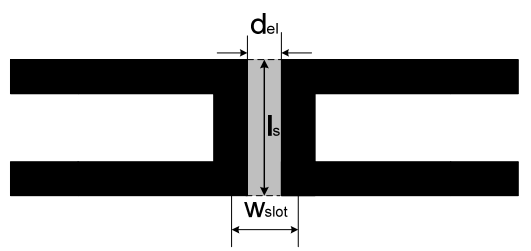

Fig. 1(b). Magnetic coupling configuration.

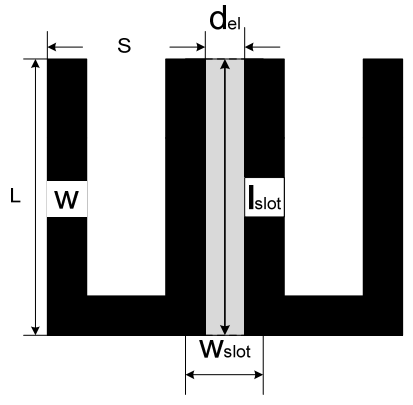

Fig.1(c). Mixed coupling configuration.

Typical input/output (I/O) coupling structures for coupled microstrip resonator filters, namely the coupled line structures, are shown with the microstrip open-loop resonator. The coupling of the coupled line stricture in Fig. 2 can be found from the coupling gap $g$ and the line width w. Normally, a smaller gap and a narrower line result in a stronger I/O coupling or a smaller external quality factor of the resonator.

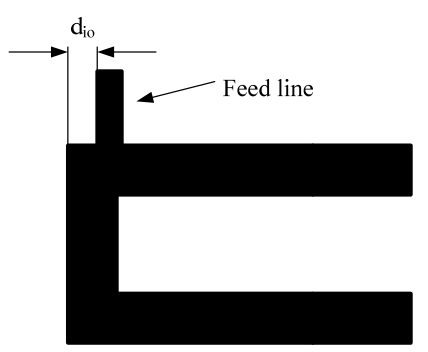

Fig. 2. The coupling of a resonator with its feed line.

The design parameters of bandpass filters, i.e., the coupling coefficients and external quality factor in Fig. 3, can be determined in terms of circuit elements of a lowpass prototype filter of Fig. 4, which consists of lumped capacitors and ideal admit The design parameters of bandpass filters, i.e., the coupling coefficients and external quality factor in Fig. 3, can be determined in terms of circuit elements of a low-pass prototype filter of Fig. 4, which consists of lumped capacitors and ideal admittance inverters. The relationships between the bandpass design parameters and the low-pass elements are

$$
\begin{gathered}
Q_{e i}=Q_{e o}=\frac{C_{1}}{F B W} \\
M_{n, n-1}=M_{N-n, N-n+1} \\
=\frac{F B W}{\sqrt{C_{n} C_{n+1}}}, \text { for } \mathrm{n}=1 \text { to } \frac{N}{2} \\
M_{m, m+1}=\frac{F B W \cdot J_{m}}{C_{m}}, \text { for } \mathrm{m}=\frac{N}{2} \\
M_{m-1, m+2}=\frac{F B W \cdot J_{m-1}}{C_{m-1}}, \text { for } \mathrm{m}=\frac{N}{2}
\end{gathered}
$$

where denotes the fractional bandwidth of the bandpass filter, is the capacitance of the lumped capacitor and is the characteristic admittance of the inverter, and is the degree of the filter. To find the element values of a low-pass prototype, one may use an approximate synthesis method described in [2]. This method is simple, but it suffers from inaccuracy and can even fail for highly selective filters that require moving the attenuation poles closer to the cutoff frequencies of the passband. However, the exact synthesis process is rather complicated and time consuming because there does not exist any closed-form formulas for the element values. The values of the attenuation pole frequency were chosen such that they 
cover a wide range of practical designs for selective microstrip bandpass filter responses. Referring to Fig. 1, the side lobe at the stopband would be too high if is smaller than the given values.

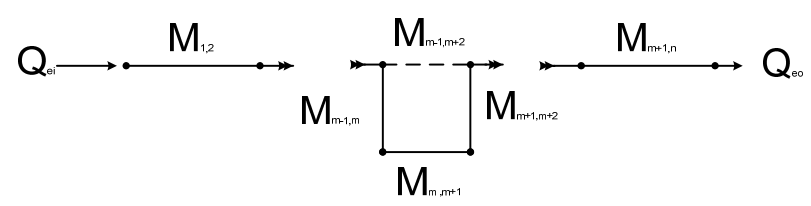

Fig. 3. General coupling structure of the bandpass filter with a single pair of finite-frequency zeros.

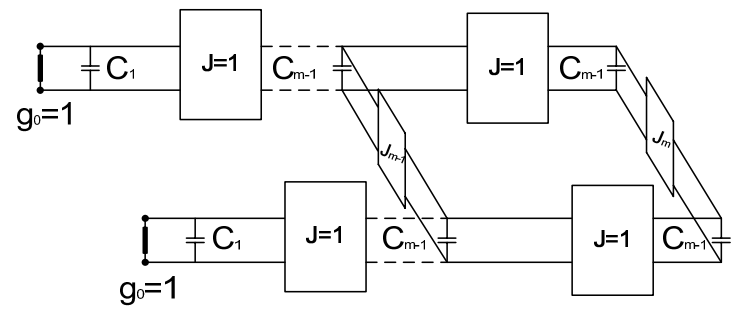

Fig. 4. Lowpass prototype filter for the filter synthesis

\section{COUPLING COEFFICIENTS}

The frequency responses of the coupling structures were obtained by using a method of moments (MoM) commercial simulation software [10]. The coupling coefficient was calculated from the two split-resonance frequencies [11]. The cross-coupled structure provides electric, magnetic, and mixed coupling. Using a parameter-extraction technique, we carry out EMsimulations to extract the external $\mathrm{Q}$ and coupling coefficient $M$ against the physical dimensions. The coupling coefficient $\mathrm{M}_{\mathrm{ij}}$ of each pair of coupled resonators can be calculated by using the equation as follows:

$$
M_{i j}=\frac{f_{2}^{2}-f_{1}^{2}}{f_{2}^{2}+f_{1}^{2}}
$$

where, $f_{1}$ and $f_{2}$ are the lower and higher split resonant frequencies. Therefore, the design curves for these coupling structures can be presented and shown in Fig. 1. The external Q of such feed structure can be characterized by the following:

$$
Q=\frac{f_{0}}{\Delta f_{3 d B}}
$$

where, $f_{0}$ is the resonant frequency of the resonator and $\Delta \mathrm{f}_{3 \mathrm{~dB}}$ is the $3 \mathrm{~dB}$ bandwidth. The design curve of the feed location on the resonator for a given external $\mathrm{Q}$ can be obtained by using EM simulation tool Sonnet. By using the design curves of the coupling coefficient and the external Q, this filter can be realized.

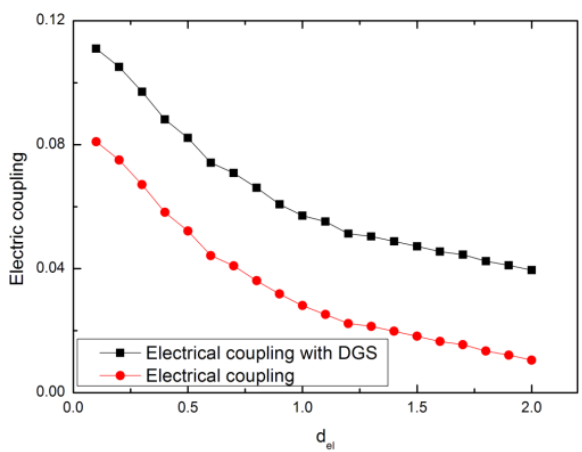

Fig. 5(a). Electric coupling coefficient.

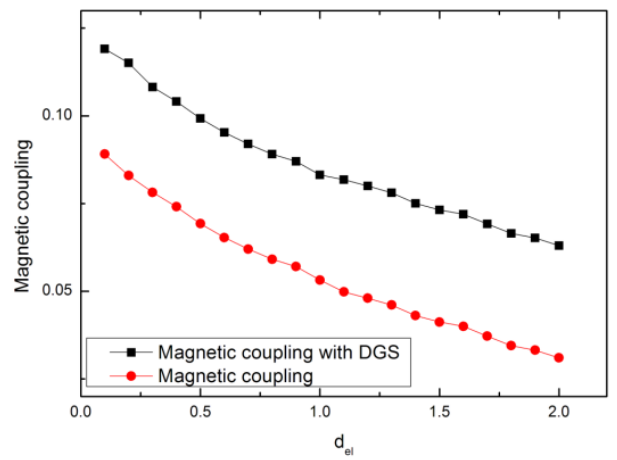

Fig. 5(b). Magnetic coupling coefficient.

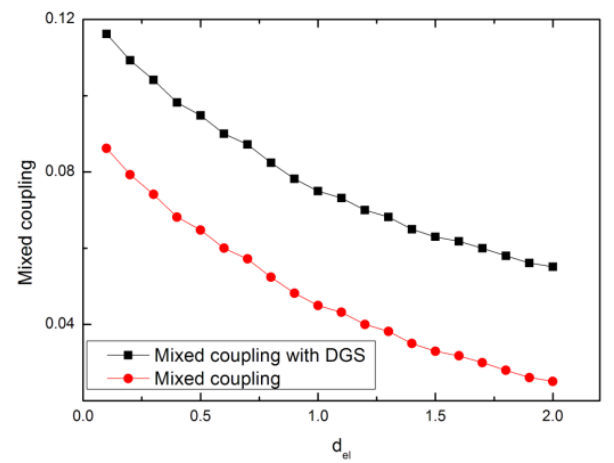

Fig. 5(c). Mixed coupling coefficient.

\section{FILTERS DESIGN AND SIMULATION}

A four-pole elliptical bandpass filter response can be implemented using the cross coupling between nonadjacent resonators. The cross-couplings give the input signal two paths from the input port to the output. The magnitude and phase of the signal are changed differently though different paths. As mentioned above, 
the multipath effect may cause attenuation poles at finite frequencies if the couplings among the resonators are properly designed. Fig. 6 shows the four-pole elliptic function bandpass filter with using miniaturized hairpin resonators. In the configuration, significant couplings exist between any two neighboring miniaturized hairpin resonators. The structure can be extended to form crosscoupled filters of higher orders. Cross-coupled bandpass filters with using compact hairpin resonators are designed to have a fractional bandwidth of $100 \mathrm{MHz}$ at a mid-band frequency $f_{0} 1.8 \mathrm{GHz}$. The filter was fabricated on Teflon substrate. The relationships between the bandpass design parameters and coupling coefficient are as follows [14]: $\mathrm{K}_{12}=0.0322, \mathrm{~K}_{23}=$ $0.0393, \mathrm{~K}_{34}=0.03173$ and $\mathrm{K}_{41}=0.030$.

The spacing designated in the cross-coupled filter is as follows: $\mathrm{w}_{1}=1.1 \mathrm{~mm}, \mathrm{w}_{2}=2.4 \mathrm{~mm}, \mathrm{w}_{3}=5.8 \mathrm{~mm}, \mathrm{w}_{4}=$ $1.9 \mathrm{~mm}, \mathrm{w}_{5}=1.2 \mathrm{~mm}$ and $\mathrm{w}_{6}=0.6 \mathrm{~mm}$. The effects on the center frequency and bandwidth are negligible as long as the gap tuning is small. The measured passband insertion loss is $-3 \mathrm{~dB}$, good agreement with the simulation. Fig. 7 shows the simulated and measured characteristics of the filters. Both filters exhibit a good rejection at second harmonic as predicted. Fig. 8 shows the prototype of bandpass filter.

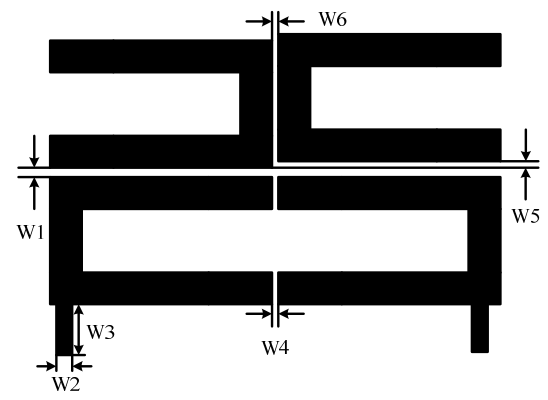

Fig. 6. Layout of the microstrip four-pole elliptic function compact filer.

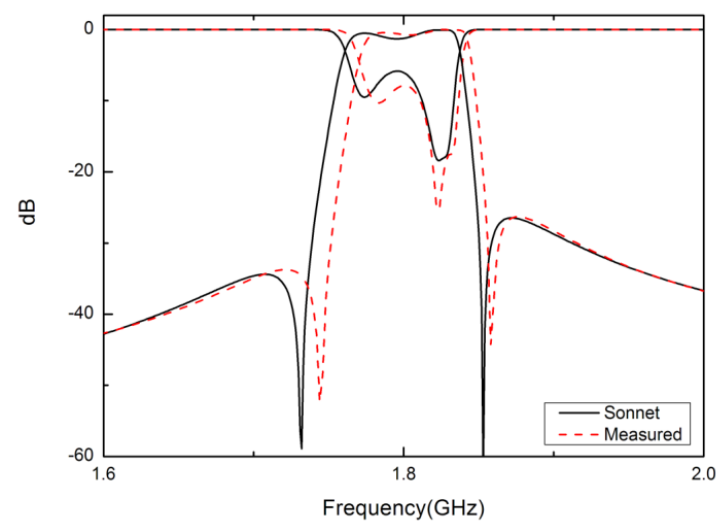

Fig. 7. Simulation and measured results of BPF.

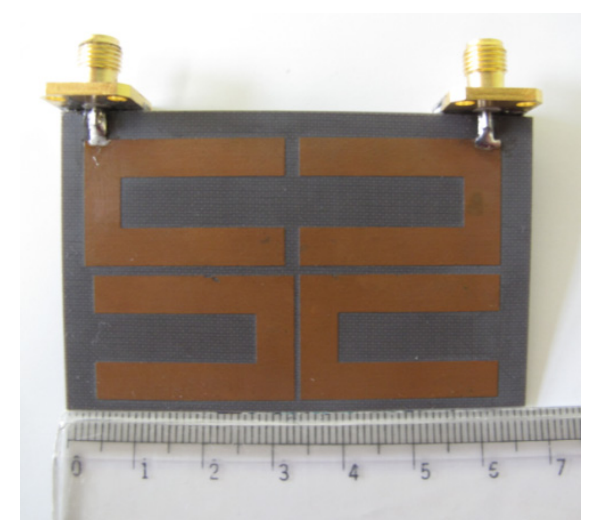

Fig. 8. Fabricated prototype.

\section{BANDPASS FILTERS WITH DGS DESIGN AND SIMULATION}

Based on the above results, some 4-pole cross-coupled planar microwave BPFs with a single or with two ground slots were designed. The slots are chosen in the center of ground plane $(9 \mathrm{~mm} \times 3 \mathrm{~mm})$. Fig. 9 shows the layout of the microstrip four-pole elliptic function compact filer with DGS. Fig. 10 shows the simulated and measured characteristics of the filters. Fig. 11 shows the top plane of proposed structure, and Fig. 12 shows the ground plane of proposed structure. Both filters exhibit a good rejection at second harmonic as predicted. But the spacing is changed. The spacing is as follows: $\mathrm{W}_{1}=1.2 \mathrm{~mm}, \mathrm{~W}_{2}=$ $2.4 \mathrm{~mm}, \mathrm{~W}_{3}=5.8 \mathrm{~mm}, \mathrm{~W}_{4}=1.9 \mathrm{~mm}, \mathrm{~W}_{5}=1.2 \mathrm{~mm}$ and $\mathrm{W}_{6}=1.2 \mathrm{~mm}$. Fig. 10 shows the prototype of bandpass filter with DGS.

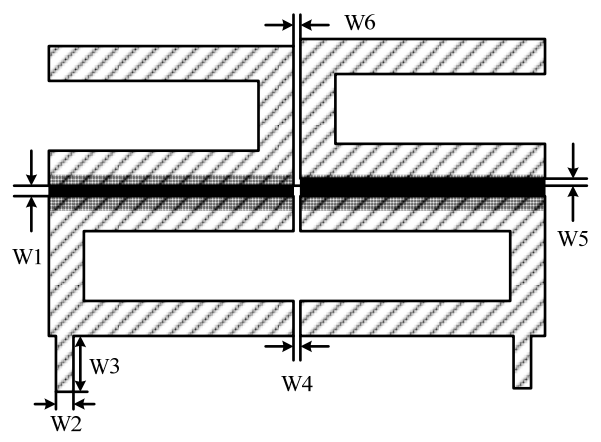

Fig. 9. Layout of the microstrip four-pole elliptic function compact filer with DGS. 


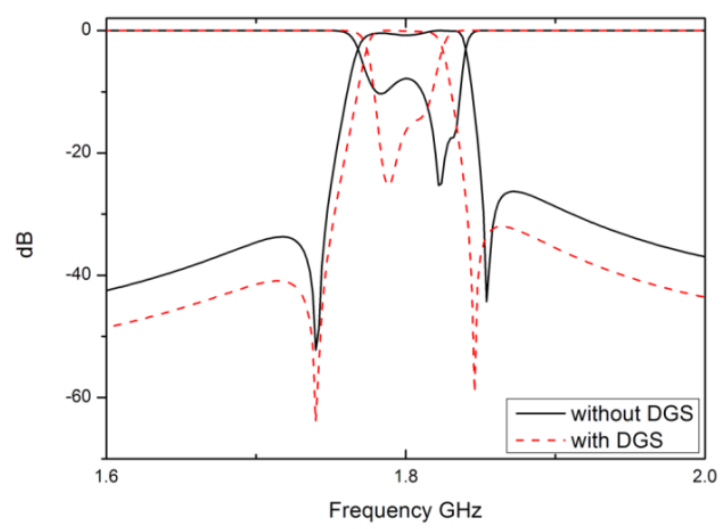

Fig. 10. Compare of simulation results of BPF.

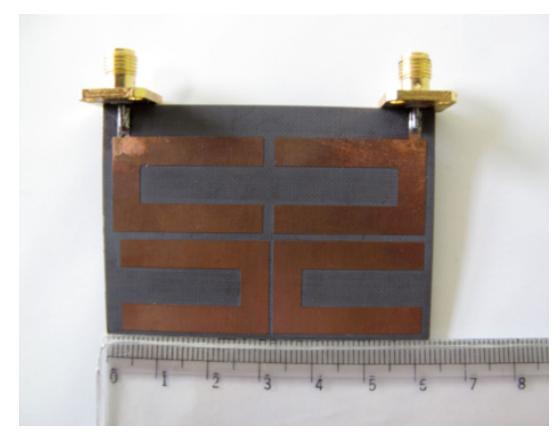

Fig. 11. Fabricated prototype of bandpass filer with DGStop layer.

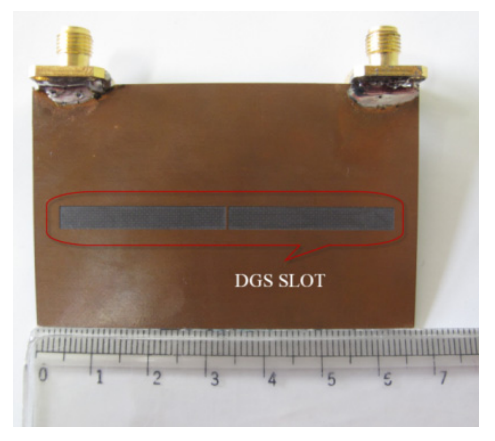

Fig. 12. Fabricated prototype of bandpass filer with DGS bottom layer

\section{CONCLUSIONS}

The main advantage of the BPF with ground slots stays in the possibility of using larger gaps between resonators. This solution is especially convenient when tight couplings are needed. The filters' layouts were designed after a study of the coupling coefficients versus gaps, based on EM-field simulation.

The designed filters with ground slots under the mixed coupling were fabricated and tested. The measured frequency responses of these proposed filter structures demonstrated the validity of the design and of the EM- field simulations. The DGS design can be also applied to many other types of bandpass filters, allowing a relaxation of the fabrication tolerances. If better performances are required, lower loss substrates should be used.

\section{REFERENCES}

[1] S.B. Cohn, "Parallel-coupled transmission line resonator filters", IEEE Trans. Microwave Theory Tech., vol. 6, pp. 223-231, 1958.

[2] S. Caspi and J. Adelmam, "Design of combline and interdigital filter with tapped-line input", IEEE Trans. Microwave Theory Tech., vol. 36, pp.759-763, 1988.

[3] G.L. Matthaei, "Comb-line bandpass filter of narrow or moderate bandwidth", IEEE Trans. Microwave Theory Tech., vol. 6, pp. 8291, 1963.

[4] U.H. Gysel, "New theory and design for hairpin-line filters", IEEE Trans. Microwave Theory Tech., vol. 22, pp. 523-531, 1974.

[5] E.G. Cristal and S. Frankel, "Hairpin-line and hybrid hairpinline/half-wave parallel-coupled-line filters", IEEE Trans. Microwave Theory Tech., vol. 20, pp. 719-728, 1972.

[6] J.S. Hong and M.J. Lancaster, "Cross-coupled microstrip hairpinresonator filters", IEEE Trans. Microwave Theory Tech., vol. 46, pp. $118-122.1998$

[7] G.L. Matthaei, N.O. Fenzi, R.J. Forse, and S.M. Rohlfing, "Hairpin combfilters for HTS and other narrow-band applications", IEEE Trans. Microwave Theory Tech. vol. 45 (1997), 1226-1231.

[8] RAO Q., JOHNSTON R. H., "Modified Aperture Coupled Microstrip Antenna", IEEE Trans. Antennas Propagat., vol. 52, no. 12, pp. 3397-3401, 2004.

[9] KIM J. P., PARK W. S., "An Improved Network Modelling of Slot-Coupled Microstrip Lines", IEEE Trans. Microwave Theory and Tech., vol. 46, no. 10, pp. 1484-1491, 1998

[10] User's Manual, Sonnet Software, Inc., New York - Sonnet Professional, version 10.52, 2005.

[11] HONG J.S., LANCASTER M.J., "Couplings of Microstrip Square Open-Loop Resonators for Cross-Coupled Planar Microwave Filters", IEEE Trans. Microwave Theory and Tech., vol. 44, pp. 2099-2109, 1996.

[12] CAMERON R., "Advanced Coupling Matrix Synthesis Techniques for Microwave Filters", IEEE Trans. Microwave Theory and Tech., vol. 51, 2003.

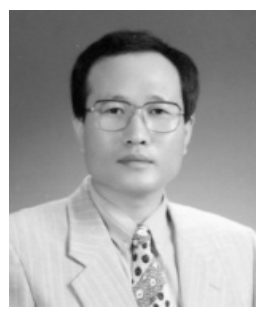

Gi-Rae Kim received the B.S., M.S. in Electronic Engineering from the Sogang University, Seoul, Korea, in 1986, 1988, respectively. He also received Ph.D. from Kyungnam University, Korea in 1999. From 1988 to 1993 he was a researcher in Communication Research Center of Samsung Electronics Co. Ltd. Since 1999, he has been on the faculty of Electronics Engineering Department at the Silla University, Busan, Korea. 